ITEP-TH-44/02

math.AG/0209145

\title{
R-Matrix Structure of Hitchin System in Tyurin Parameterization
}

\author{
V.A. Dolgushev ${ }^{1}$ \\ Department of Mathematics, MIT, \\ 77 Massachusetts Avenue, \\ Cambridge, MA, USA 02139-430\%; \\ University Center, JINR, Dubna, \\ 141 980, Moscow Region, Russia; \\ Institute for Theoretical and Experimental Physics, \\ 117259, Moscow, Russia.
}

\begin{abstract}
We present a classical $r$-matrix for the Hitchin system without marked points on an arbitrary non-degenerate algebraic curve of genus $g \geq 2$ using Tyurin parameterization of holomorphic vector bundles.
\end{abstract}

\section{Introduction}

The study of the moduli space of holomorphic vector bundles over an algebraic curve motivated by the geometric Langlands conjecture [4] is now one of the most fascinating topics of modern algebraic geometry. Important tools for the investigation are integrable systems of Hitchin type [20], [21], [24], [25], [26] whose configuration spaces are defined as connected components of the moduli space of holomorphic vector bundles over compact Riemann surfaces.

It is not an easy task to give a satisfactory description of a Hitchin system since its definition is implicit and at the first sight it is clear neither how to find its Lax representation nor how to write down the respective equations of motion. For the case of algebraic curves of genus zero and one this question was solved in papers [7], [14], [16], [26] and for Schottky curves of an arbitrary higher genus a description of Hitchin systems was proposed in [9].

In the paper by A. Tyurin [34] a classification of holomorphic vector bundles over algebraic curves of arbitrary genus is obtained and a convenient parameterization of big cells of connected components of the moduli space of the bundles is suggested. After its introduction in [34] Tyurin parameterization is used in the works on integrable differential systems [8], [19], [21], [22], [28] and two papers [8], [21] are worthy of mention, in which the Tyurin description is used to parameterize Hitchin systems.

\footnotetext{
${ }^{1}$ E-mail: vald@mit.edu
} 
In [8] the parameterization of Hitchin systems is obtained for the case of rank 2 holomorphic vector bundles of degree $2 g$ over algebraic curves of genus $g \geq 1$ and in [21] Tyurin description is used to parameterize an arbitrary Hitchin system and to construct infinite-dimensional field analogues ${ }^{2}$ of the systems of Hitchin type. The results of the papers [8] and [21] show that Tyurin parameterization should enable us to achieve a rough but explicit description of quantum Hitchin systems, and the first step in this direction is a construction of classical $r$-matrix structures for Hitchin systems which will allow us to quantize the systems in a quantum group theoretic setting [10], [15], [18].

The concept of a classical $r$-matrix was originally introduced in works of "Leningrad school" [30], [32] (see also book [12]) as a natural object that encodes the Hamiltonian structure of the Lax equation, provides the involution of integrals of motion [3], and gives a natural framework for quantizing integrable systems.

In this paper we present a classical $r$-matrix for the Hitchin system without marked points on an arbitrary non-degenerate algebraic curve of genus $g \geq 2$ using Tyurin parameterization of the moduli space of rank $n$ holomorphic vector bundles of degree $n g$.

Following Tyurin [34] a generic holomorphic vector bundle $\mathcal{B}$ of this type over a non-degenerate curve $\Sigma$ has an $n$-dimensional space $H^{0}(\Sigma, \mathcal{B})$ of holomorphic sections and for a generic point $P$ of the curve $\Sigma$ the sections generate a basis in the respective fiber $\mathcal{B}_{P}$. However, the evaluations of these sections on $n g$ points $\gamma_{a} \in \Sigma, a=1, \ldots, n g$ are linearly dependent and for distinct points $\gamma_{a}$ they determine subspaces $V_{a} \in \mathcal{B}_{\gamma_{a}}$ of codimension 1 or just one-dimensional linear subspaces $l_{a}$ in the dual space $H^{0}(\Sigma, \mathcal{B})^{*}$. The collection of lines $l_{a} \in H^{0}(\Sigma, \mathcal{B})^{*}$ can be identified with nonzero vectors $\alpha_{a} \in \mathbb{C}^{n}$, which are defined up to the scalar multiples

$$
\alpha_{a} \mapsto \lambda_{a} \alpha_{a}, \quad \lambda_{a} \in \mathbb{C}, \lambda_{a} \neq 0
$$

and up to the following transformations of the group $S L_{n}(\mathbb{C})^{3}$

$$
\alpha_{a}^{i} \mapsto \tilde{\alpha}_{a}^{i}=\alpha_{a}^{j}\left(G^{-1}\right)_{i}^{j}, \quad \operatorname{det}\left\|G_{j}^{i}\right\|=1, i, j=1, \ldots n,
$$

generated by changes of basis in $H^{0}(\Sigma, \mathcal{B})$.

Thus, we arrive at the Tyurin map from an open dense set of the moduli space of rank $n$ holomorphic vector bundles of degree $n g$ over the curve $\Sigma$ to the following quotient

$$
\left[\Sigma \times \mathbb{P}\left(\mathbb{C}^{n}\right)\right]^{(n g)} / S L_{n}(\mathbb{C})
$$

where the notation ${ }^{(n g)}$ stands for the symmetric direct product.

The set of points $\gamma_{a} \in \Sigma$ and vectors $\alpha_{a} \in \mathbb{C}^{n}$ are referred to as Tyurin parameters, and the main statement of the paper [34] we are going to use is that an open dense set of the moduli space of rank $n$ holomorphic vector bundles of degree $n g$ over the curve $\Sigma$ is parameterized by points of the quotient (2).

\footnotetext{
${ }^{2}$ In this context paper [24] is also worthy of mention, in which the case of two-dimensional version of the elliptic Gaudin system is considered in detail.

${ }^{3} \mathrm{We}$ assume here the summation over repeated indices.
} 
Note that in our considerations we are not going to bother about the singularities of the quotient of the space $\left[\Sigma \times \mathbb{P}\left(\mathbb{C}^{n}\right)\right]^{n g}$ with respect to the action of the symmetric group $S_{n g}$ and in what follows we omit factorization of our phase space with respect to permutations.

In order to parameterize the phase space of the Hitchin system without marked points one has to supplement Tyurin parameters $\left(\gamma_{a}, \alpha_{a}\right)$ with points $\kappa_{a} \in T_{\gamma_{a}}^{*} \Sigma$ and vectors $\beta_{a} \in \mathbb{C}^{n}$, which are subject to the following conditions

$$
\begin{gathered}
\sum_{i=1}^{n} \beta_{a}^{i} \alpha_{a}^{i}=0, \\
T_{i j}=\sum_{a=1}^{n g} \beta_{a}^{i} \alpha_{a}^{j}=0 .
\end{gathered}
$$

Equation (3) means that the $\beta_{a}^{i}$ may be regarded as coordinates in the cotangent space $T_{\alpha_{a}}^{*} \mathbb{P}^{n-1}$ and equations (4) are just the first class constraint conditions corresponding to the symplectic action of the group $S L_{n}(\mathbb{C})$ on the parameters $\alpha_{a}^{i}$ and $\beta_{a}^{i}$, that is

$$
\alpha_{a}^{i} \mapsto \alpha_{a}^{j}\left(G^{-1}\right)_{i}^{j}, \quad \beta_{a}^{i} \mapsto G_{j}^{i} \beta_{a}^{j}, \quad\left\|G_{j}^{i}\right\| \in S L_{n}(\mathbb{C}) .
$$

In other words, the phase space of the Hitchin system in question can obtained via symplectic reduction in the space

$$
\mathcal{P}=T^{*}\left[\Sigma \times \mathbb{P}\left(\mathbb{C}^{n}\right)\right]^{n g}
$$

on the surface of the first class constraints ${ }^{4}(4)$.

The main statement of the paper (see Theorem 1) is that the Krichever Lax matrix of the Hitchin system being extended to the symplectic manifold (6) admits a simple $r$-matrix structure, which is defined by a matrix-valued meromorphic section of the bundle $\Sigma \times T^{*} \Sigma$ over the direct product of curves $\Sigma \times \Sigma$. We argue that using the $r$-matrix structure one can easily derive the classical $r$-matrix for the initial Lax matrix of the Hitchin system either with a help of a gauge invariant extension of the Krichever Lax matrix to the manifold (6) or with the help of on-shell Dirac brackets between the entries of the initial extension of the Krichever Lax matrix.

Note however that the $r$-matrix structure of the extended system is much simpler than the resulting $r$-matrix of the Hitchin system and this remarkable simplification turns out to be possible due to the fact ${ }^{5}$ that the Krichever Lax matrix of the Hitchin system, being a meromorphic differential on the curve $\Sigma$, can be extended to the symplectic manifold (6) in such a way that the extension is also a meromorphic matrix-valued differential on $\Sigma$.

The organization of the paper is as follows. In the second section we present the extension of the Krichever Lax matrix for the Hitchin system without marked points on a non-degenerate algebraic curve of genus $g \geq 2$ and propose that the extended

\footnotetext{
${ }^{4} \mathrm{~A}$ similar trick is used in [7] for description of Hitchin systems associated with marked rational and elliptic curves.

${ }^{5} \mathrm{I}$ am indebted to A.M. Levin for the technical trick concerning the extension of the Krichever Lax matrix.
} 
system admits an $r$-matrix structure, which is defined as a meromorphic matrixvalued function on one copy of the curve and a meromorphic 1-form on another copy of the same curve. Then, postponing the proof of this proposition to the next section, we show how to derive the classical $r$-matrix for the genuine Krichever Lax matrix of the Hitchin system using the above $r$-matrix structure.

Before presenting the proof in section 3 we show that a matrix-valued differential that enters into the definition of the above $r$-matrix structure does exist. We also give the properties of the differential as a function in the first variable and identify derivatives of the extended Krichever Lax matrix with respect to phase space variables as meromorphic differentials on the algebraic curve.

In the concluding section we mention dynamical properties of the presented $r$ matrices, discuss a possibility to derive the $r$-matrices using an infinite-dimensional Hamiltonian reduction, and raise some other questions.

In the appendix at the end of the paper we present the Krichever lemma, which is used throughout the paper as a tool, that enables us to identify meromorphic vectorvalued differentials by their singular parts and certain linear equations for their regular parts. Although the statement is analogous to Lemma 2.2 in [21] we present its proof in the appendix since in some respect the statement generalizes the lemma and the presented proof differs from the one given in [21].

In this paper we use standard notations for Poisson brackets between entries of a Lax matrix. For example, if $L(z)$ is a matrix-valued function and

$$
r(z, w)=\sum_{i, j, k, l} r_{i j k l}(z, w) e_{i j} \otimes e_{k l}
$$

where

$$
\left(e_{i j}\right)_{k l}=\delta_{i k} \delta_{j l}
$$

are the elements of the standard basis in $g l_{n}(\mathbb{C})$ then the expression

$$
\left\{L_{1}(z), L_{2}(w)\right\}=\left[r(z, w), L_{1}(z)\right]-\left[r_{21}(w, z), L_{2}(w)\right]
$$

means that Poisson brackets between the entries $L_{i j}(z)$ and $L_{k l}(w)$ take the following form

$$
\begin{gathered}
\left\{L_{i j}(z), L_{k l}(w)\right\}=\sum_{m=1}^{n}\left(r_{i m k l}(z, w) L_{m j}(z)-L_{i m}(z) r_{m j k l}(z, w)\right)- \\
-\sum_{m=1}^{n}\left(r_{k m i j}(w, z) L_{m l}(w)-L_{k m}(w) r_{m l i j}(w, z)\right) .
\end{gathered}
$$

Throughout the paper we assume that $\Sigma$ is a non-degenerate algebraic curve of genus $g \geq 2$.

\section{$2 \quad R$-matrix structure for the Hitchin system with- out marked points.}

We start with the following particular case of Lemma 2.2 in [21] 
Lemma 1 For a generic set of pairs $\left(\gamma_{a}, k_{a}\right), \gamma_{a} \in \Sigma, k_{a} \in T_{\gamma_{a}}^{*} \Sigma, a=1, \ldots, n g$ and complex parameters $\alpha_{a}^{i}$ and $\beta_{a}^{i} i=1, \ldots n$ such that

$$
\sum_{i=1}^{n} \beta_{a}^{i} \alpha_{a}^{i}=0
$$

there exists a unique matrix-valued meromorphic differential $L_{i j}=L_{i j}(z) d z$ of the third kind satisfying the following properties

1. The differential $L_{i j}$ has poles only at the points $\gamma_{a}$ and at some fixed point $P \in \Sigma$.

2. On a neighborhood of the point $\gamma_{a}$ the differential $L_{i j}(z) d z$ behaves like

$$
L_{i j}(z)=\frac{\beta_{a}^{i} \alpha_{a}^{j}}{z-z\left(\gamma_{a}\right)}+L_{i j}^{a, 0}+L_{i j}^{a, 1}\left(z-z\left(\gamma_{a}\right)\right)+\ldots
$$

3. $\alpha_{a}$ is a left eigenvector for the matrix $\left\|L_{i j}^{a, 0}\right\|$ with the eigenvalue $\kappa_{a}$

$$
\sum_{i=1}^{n} \alpha_{a}^{i} L_{i j}^{a, 0}=\kappa_{a} \alpha_{a}^{j}
$$

The differential $L_{i j}(z) d z$ is obviously invariant under the transformations

$$
\alpha_{a} \mapsto \lambda_{a} \alpha_{a}, \quad \beta_{a} \mapsto \lambda_{a}^{-1} \beta_{a}, \quad \lambda_{a} \in \mathbb{C}, \quad \lambda_{a} \neq 0
$$

and, hence, it may be regarded as a function with values in meromorphic differentials on an open dense set of the space (6) so that the components of the vector $\alpha_{a}$ are identified with homogeneous coordinates in $\mathbb{P}\left(\mathbb{C}^{n}\right)$ and the components of the vector $\beta_{a}$, being subject to the conditions (8) define a point in the respective cotangent space $T_{\alpha_{a}}^{*} \mathbb{P}\left(\mathbb{C}^{n}\right)$.

The differential $L_{i j}(z) d z$ and its natural generalizations were originally found in the paper [21] by Krichever as solutions of the momentum map equations for Hitchin systems. Although the differential $L_{i j}(z) d z$ is not a Krichever Lax matrix of the Hitchin system without marked points since equations (4) are not imposed, $L_{i j}(z) d z$ may be regarded as an extension of the above Lax matrix to the symplectic manifold $\mathcal{P}$. In what follows, we refer to $L$ as a Krichever Lax differential.

Notice that in view of Lemma 2.1 of [21], the differential $L_{i j}(z) d z$ can be identified with a meromorphic section with a single pole at the point $P$ of the bundle $\operatorname{End}(\mathcal{B}) \otimes \mathcal{K}$ where $\mathcal{B}$ is the holomorphic bundle over $\Sigma$ corresponding to the Tyurin parameters $\gamma_{a}$ and $\alpha_{a}^{i}$ and $\mathcal{K}$ is a canonical bundle of the curve $\Sigma$.

Soon we will show that the Krichever Lax differential being considered as a function on the symplectic manifold $\mathcal{P}$ admits an $r$-matrix structure, but now we present an important ingredient which enters into the definition of the $r$-matrix structure in question.

Lemma 2 For a generic set of Tyurin parameters $\alpha_{a}$ and $\gamma_{a}$ there exists a unique matrix-valued differential $r_{j k}(z, w) d w$ such that 
1. $r_{j k}(z, w) d w$ is a meromorphic function in $z$ and a meromorphic 1-form in $w$,

2. $r_{j k}(z, w) d w$ is holomorphic in $w$ everywhere on $\Sigma$ except the points $w=w(P)$ and $w=z$, where it has simple poles with residues $\delta_{j k}$ and $-\delta_{j k}$, respectively,

3. $\alpha_{a}$ are null vectors for the matrices $r_{j k}\left(z, \gamma_{a}\right)$

$$
\sum_{k=1}^{n} r_{j k}\left(z, \gamma_{a}\right) \alpha_{a}^{k}=0
$$

The existence of the meromorphic differential $r_{j k}(z, w) d w$ which is also a meromorphic function in $z$ satisfying the above conditions is proved in subsection 3.1 where a stronger statement (see Lemma 3 ) concerning the properties of the differential $r_{j k}(z, w) d w$ as a function in $z$ is also formulated. To this end, the uniqueness of the differential $r_{j k}(z, w) d w$ follows directly from the Krichever lemma.

We now present the main statement of the paper.

Theorem 1 For an arbitrary non-degenerate algebraic curve $\Sigma$ of genus $g \geq 2$ the canonical Poisson brackets of the space (6) between the entries of the Krichever Lax differential (9) obey the Yang-Baxter relation

$$
\left\{L_{1}(z), L_{2}(w)\right\} d z \otimes d w=\left[r(z, w), L_{1}(z)\right] d z \otimes d w-\left[r_{21}(w, z), L_{2}(w)\right] d z \otimes d w,
$$

where the differential $r(z, w) d w$ is given by the formula

$$
r(z, w) d w=\sum_{i, j, k} r_{j k}(z, w) e_{i j} \otimes e_{k i} d w,
$$

and $r_{j k}(z, w) d w$ is the meromorphic 1-form defined in Lemma 2.

We will refer to the differential (14) as an $r$-matrix differential.

In the following section we present an algebraic-geometric proof of the theorem. First we explain how to achieve the $r$-matrix for the Hitchin system we consider using the differential (14).

As we have mentioned in the introduction, the phase space of the Hitchin system without marked points can be identified with an open dense set of the quotient of the constraint surface (4) in the space (6) with respect to the symplectic action (5) of the group $S L_{n}(\mathbb{C})$.

In other words, if one chooses some gauge fixing conditions

$$
\chi^{i j}\left(\alpha_{a}^{k}\right)=0
$$

for the transformations (5) then the phase space of the Hitchin system can be roughly identified with an intersection of the surfaces (4) and (15) in the space (6) and the respective Krichever Lax matrix is defined as the differential (9), restricted to the intersection

$$
l_{i j}(z) d z=\left.L_{i j}(z) d z\right|_{T_{k l}=\chi^{k l}=0} .
$$


Obviously, the Lax matrix (16) is a meromorphic differential on the curve $\Sigma$ with the same properties (9), (10) as the Krichever Lax differential except that the point $P$ is now regular for the differential (16). In view of Lemma 2.1 of [21], this means that the differential (16) can be identified with a holomorphic section of the bundle $\operatorname{End}(\mathcal{B}) \otimes \mathcal{K}$.

The gauge transformations (5) of the Krichever Lax differential (9) have a form of adjoint action

$$
L(z) \mapsto G L(z) G^{-1}, \quad G \in S L_{n}(\mathbb{C}),
$$

and hence, whatever gauge fixing conditions are chosen, the desired $r$-matrix of the Hitchin system can be derived from the $r$-matrix differential (14) either with the help of a gauge invariant extension of the Krichever Lax matrix (16) or with the help of on-shell Dirac brackets between the entries of the differential (9).

Recall that the gauge invariant extension of Lax matrices was originally used to calculate classical $r$-matrices for integrable systems in the works [1] and [2]. In a more general situation Dirac brackets and gauge invariant extension of Lax matrices are used for analogous calculations in the paper [5]. At last, in the paper [13] Dirac bracket technique is used in a specific framework to obtain new examples of EtingofVarchenko dynamical $r$-matrices [11].

To derive the classical $r$-matrix for the Hitchin system we use the gauge invariant extension of the Krichever Lax matrix (16) to the space (6). For example, if some $n \times n$-minor $\left\|\alpha_{a_{j}}^{i}\right\|$ of the matrix $\left\|\alpha_{a}^{i}\right\|$ where $1 \leq a_{1}<a_{2}<\ldots<a_{n} \leq n g$ is non-degenerate we can choose gauge fixing conditions in the form [21]

$$
\alpha_{a_{j}}^{i}=0, i \neq j, \quad \alpha_{b}^{1}=\alpha_{b}^{2}=\ldots=\alpha_{b}^{n}
$$

where $b$ does not coincide with any of the indices $a_{1}, a_{2}, \ldots a_{n}$.

On an open region of the space (6) one can define the $S L_{n}$-valued function $G\left(\alpha_{a}\right)$ such that if the vectors $\alpha_{a}$ do not satisfy the gauge fixing conditions (18) then the transformed vectors $\tilde{\alpha}_{a}$

$$
\tilde{\alpha}_{a}^{i}=\alpha_{a}^{j}\left(G^{-1}\left(\alpha_{c}\right)\right)_{i}^{j}
$$

do so. Otherwise $G\left(\alpha_{a}\right)$ is just the identity matrix.

Then the matrix-valued differential

$$
l^{G}(z)=G\left(\alpha_{a}\right) L(z) G^{-1}\left(\alpha_{a}\right)
$$

turns out to be a desired gauge invariant extension of the Krichever Lax matrix (16) to the space $\mathcal{P}$ and the $r$-matrix in question takes the form

$$
r^{H}(z, w) d w=\left.\left(r(z, w) d w+\left\{G_{1}\left(\alpha_{a}\right), L_{2}(w)\right\} d w\right)\right|_{\text {on shell }},
$$

where the notation $\left.\right|_{\text {on shell }}$ means that the expression in the parenthesis is considered on the surface of the constraints (4) and (18).

Example. Although Hitchin systems without marked points are non-trivial only for algebraic curves of genus $g \geq 2$ the Krichever Lax differential (9) and its $r$-matrix structure (14) exist on an elliptic curve as well. 
To show this, we realize an elliptic curve $\Gamma$ as a quotient $\Gamma=\mathbb{C} /\{1, \tau\}, \operatorname{Im} \tau>0$ and denote the parameters $\gamma_{a}$ and $k_{a}$ by $q_{a}$ and $p_{a}$, respectively, where $a$ now runs from 1 to $n$. Then, the Krichever Lax differential (9) and the $r$-matrix differential (14) can be written in terms of the standard $\theta$-function as follows

$$
\begin{gathered}
L_{i j}(z)=\sum_{k, l=1}^{n} \pi_{i}^{k} \tilde{L}_{k l}(z) \alpha_{l}^{j}, \quad \tilde{L}_{i i}=p_{i}, \\
\tilde{L}_{i j}(z)=\sum_{k=1}^{n} \alpha_{i}^{k} \beta_{j}^{k} \frac{\theta\left(z-q_{i}\right) \theta\left(z+q_{i}-q_{j}\right) \theta\left(q_{j}\right) \theta^{\prime}(0)}{\theta(z) \theta\left(z-q_{j}\right) \theta\left(q_{j}-q_{i}\right) \theta\left(q_{i}\right)}, \quad i \neq j, \\
r(z, w)=\sum_{i, j=1}^{n}(E(z-w)+E(w)) e_{i j} \otimes e_{j i}- \\
-\sum_{i, j, k, a=1}^{n} \pi_{k}^{a} \alpha_{a}^{j}\left(E\left(z-q_{a}\right)+E\left(q_{a}\right)\right) e_{i j} \otimes e_{k i},
\end{gathered}
$$

where $\left\|\pi_{i}^{j}\right\|$ is the inverse matrix to $\left\|\alpha_{k}^{l}\right\|$

$$
\begin{gathered}
\sum_{k=1}^{n} \alpha_{i}^{k} \pi_{k}^{j}=\delta_{i}^{j}, \\
\theta(z)=\sum_{m \in \mathbb{Z}} \exp \left(\pi i \tau(m+1 / 2)^{2}+2 \pi i(m+1 / 2)(z+1 / 2)\right),
\end{gathered}
$$

and

$$
E(z)=\frac{\theta^{\prime}(z)}{\theta(z)} .
$$

To explain the relation of the Lax matrix (21) to Lax representation of known integrable systems we have to enlarge the phase space parameterized by coordinates $q_{a}, p_{a}, \alpha_{a}^{i}$, and $\beta_{a}^{i}$ with some coadjoint orbit $\mathcal{O}$ of the group $S L_{N}$. Symplectic reduction of this space to the first class constraint surface

$$
\sum_{a=1}^{n g} \beta_{a}^{i} \alpha_{a}^{j}+\eta_{i j}=0
$$

leads us to the phase space and the Lax matrix ${ }^{6}$ of the elliptic spin Calogero-Moser system [26], [27]. Here $\eta_{i j}$ denote conventional coordinates on the coadjoint orbit $\mathcal{O}$. If we now restrict $\mathcal{O}$ to be the maximal coadjoint orbit we just get the particular case of one marked point of the integrable system considered in [7]. The latter system is now generally regarded as an elliptic Gaudin system [26], [33].

\footnotetext{
${ }^{6}$ We note that the Lax matrix of the elliptic spin Calogero-Moser system was originally presented as a meromorphic function on the elliptic curve in the paper [21]
} 


\section{The proof of the Yang-Baxter relation}

The proof of Theorem 1 is based on the observation that both sides of equation (13) satisfy the same properties, which, in turn, uniquely define them as meromorphic forms on the direct product of curves $\Sigma \times \Sigma$. Namely, it turns out that both sides of equation (13) have coincident singular parts while their regular parts at the points $\gamma_{a}$ obey the same linear inhomogeneous equations, which uniquely define the remaining arbitrariness in the holomorphic parts due to the Krichever lemma.

To calculate Poisson brackets between the entries of the Krichever Lax differential we choose the local chart of the space (6) where

$$
\alpha_{a}^{1}=1, \quad \beta_{a}^{1}=-\sum_{\mu=2}^{n} \beta_{a}^{\mu} \alpha_{a}^{\mu}, \quad \forall a=1, \ldots, n g .
$$

Note that although a choice of another local affine chart affects intermediate calculations the Poisson bracket

$$
\left\{L_{i j}(z), L_{k l}(w)\right\} d z \otimes d w
$$

is, in fact, "a function" on the space (6), and therefore the properties of the expression (25) as a form on product of curves $\Sigma \times \Sigma$ do not depend on the choice of local coordinates on $\mathcal{P}$.

Throughout this section we also assume that some local coordinates are chosen on neighborhoods of the points $\gamma_{a}$ on the curve $\Sigma$ and for simplicity we denote the coordinate $z\left(\gamma_{a}\right)$ by the same letter $\gamma_{a}$.

\subsection{Properties of the $r$-matrix differential as a function of the first argument.}

We start this subsection with the following

Lemma 3 The differential $r_{i j}(z, w) d w$ defined in Lemma 2 exists and is holomorphic in $z$ everywhere on $\Sigma$ except the points $\gamma_{a}$, where it has simple poles. The differential $r_{i j}(z, w) d w$ is also vanishing at the point $z=z(P)$

$$
r_{i j}(z(P), w) d w=0 .
$$

Proof. First, using the Krichever lemma we introduce auxiliary holomorphic vectorvalued differentials $u_{a i}(z) d z$, which are uniquely defined by the following properties

$$
\sum_{i=1}^{n} u_{a i}\left(\gamma_{b}\right) \alpha_{b}^{i}=\delta_{a b} .
$$

Using standard arguments based on the Kodaira-Nakano vanishing theorem and GAGA principles one can easily show that for an arbitrary point $Q \in \Sigma$ there exists a matrix-valued differential $\Delta_{i j}(z, w) d w$, which is holomorphic in $z$ on some open neighborhood $U_{Q}$ of the point $Q$ and holomorphic in $w$ everywhere on $\Sigma$ except the 
points $w=w(P)$ and $w=z$, where the differential has simple poles with residues $\delta_{i j}$ and $-\delta_{i j}$, respectively.

It is easy to see that the following matrix-valued differential

$$
r_{i j}^{U_{Q}}(z, w) d w=\Delta_{i j}(z, w) d w-\sum_{a, k} \Delta_{i k}\left(z, \gamma_{a}\right) \alpha_{a}^{k} u_{a j}(w) d w
$$

is meromorphic in $z$ on the neighborhood $U_{Q}$ and satisfies conditions 2 and 3 of Lemma 2.

Since conditions 2 and 3 of Lemma 2 uniquely determine $r_{i j}^{U_{Q}}(z, w) d w$ as a 1 -form in $w$ we can define the desired differential $r_{i j}(z, w) d w$ by its restrictions (28) to the sets $U_{Q}$.

Equation (28) also implies that the resulting differential $r_{i j}(z, w) d w$ is holomorphic in $z$ everywhere on $\Sigma$ except the points $\gamma_{a}$ and on the neighborhoods of the points the differential behaves like

$$
r_{i j}(z, w) d w=-\frac{\alpha_{a}^{i} u_{a j}(w) d w}{z-\gamma_{a}}+\text { regular terms. }
$$

Note also that as the differential $r_{i j}(z(P), w) d w$ is holomorphic in $w$ everywhere on $\Sigma$ equations (12) imply that the differential is in fact vanishing due to the Krichever lemma.

Thus, the statement is proved.

In order to prove the Yang-Baxter relation we have to identify the next two coefficients of the Laurent expansion of the differential $r_{i j}(z, w) d w$ in the first variable $z$ around a point $\gamma_{a}$. In the following lemma we identify these coefficients as meromorphic differentials on the curve $\Sigma$.

Lemma 4 The expansion coefficients $r_{i j}^{a, 0}(w) d w$ and $r_{i j}^{a, 1}(w) d w$ of the Laurent series

$$
r_{i j}(z, w) d w=-\frac{\alpha_{a}^{i} u_{a j}(w) d w}{z-\gamma_{a}}+r_{i j}^{a, 0}(w) d w+\left(z-\gamma_{a}\right) r_{i j}^{a, 1}(w) d w+o\left(z-\gamma_{a}\right)
$$

of the differential $r_{i j}(z, w) d w$ on a neighborhood $U_{\gamma_{a}}$ of a point $\gamma_{a}$ are uniquely defined by the following properties ${ }^{7}$ :

1. The 1-form $r_{i j}^{a, 0}(w) d w$ is holomorphic everywhere on $\Sigma$ except the points $P$ and $\gamma_{a}$, where it has simple poles with residues $\delta_{i j}$ and $-\delta_{i j}$, respectively.

2. For $b \neq a, \alpha_{b}$ is a null vector for the matrix $\left\|r_{i j}^{a, 0}\left(\gamma_{b}\right)\right\|$

$$
\sum_{j=1}^{n} r_{i j}^{a, 0}\left(\gamma_{b}\right) \alpha_{b}^{j}=0, \quad b \neq a,
$$

\footnotetext{
${ }^{7}$ Note that the uniqueness of the differentials $r_{i j}^{a, 0}(w) d w$ and $r_{i j}^{a, 1}(w) d w$ satisfying the presented properties follows from the Krichever lemma.
} 
and $\alpha_{a}$ is a null vector for the regular part the matrix $\left\|r_{i j}^{a, 0}(w)\right\|$ at the point $w=\gamma_{a}$

$$
\left.\sum_{j=1}^{n} r_{i j}^{a, 0}(w) \alpha_{a}^{j}\right|_{\text {regular part at } w=\gamma_{a}}=0
$$

3. The 1-form $r_{i j}^{a, 1}(w) d w$ has a single pole at the point $\gamma_{a}$ and on a neighborhood of the point it behaves like

$$
r_{i j}^{a, 1}(w)=-\frac{\delta_{i j}}{\left(w-\gamma_{a}\right)^{2}}+\text { regular terms }
$$

4. For $b \neq a, \alpha_{b}$ is a null vector for the matrix $\left\|r_{i j}^{a, 1}\left(\gamma_{b}\right)\right\|$ :

$$
\sum_{j=1}^{n} r_{i j}^{a, 1}\left(\gamma_{b}\right) \alpha_{b}^{j}=0, \quad b \neq a
$$

and $\alpha_{a}$ is a null vector for the regular part of the matrix $\left\|r_{i j}^{a, 1}(w)\right\|$ at the point $w=\gamma_{a}$ :

$$
\left.\sum_{j=1}^{n} r_{i j}^{a, 1}(w) \alpha_{a}^{j}\right|_{\text {regular part at } w=\gamma_{a}}=0
$$

Proof. Applying the properties of the 1-form $r_{i j}(z, w) d w$ (see Lemma 2) to the Laurent expansion (30) we get that outside the neighborhood $U_{\gamma_{a}}$ the differential $r_{i j}^{a, 0}(w) d w$ has only a simple pole at the point $w=w(P)$ with the residue $\delta_{i j}$, the differential $r_{i j}^{a, 1}(w) d w$ is holomorphic in the region $\Sigma \backslash U_{\gamma_{a}}$, and for $b \neq a \alpha_{b}$ is a right null vector for the matrices $\left\|r_{i j}^{a, 0}\left(\gamma_{b}\right)\right\|$ and $\left\|r_{i j}^{a, 1}\left(\gamma_{b}\right)\right\|$

$$
\sum_{j=1}^{n} r_{i j}^{a, 0}\left(\gamma_{b}\right) \alpha_{b}^{j}=0, \quad \sum_{j=1}^{n} r_{i j}^{a, 1}\left(\gamma_{b}\right) \alpha_{b}^{j}=0, \quad b \neq a
$$

The expansion (30) cannot be used for the case when $w$ is on the neighborhood $U_{\gamma_{a}}$ because $r_{i j}(z, w)$ is irregular at the point $z=w$.

In order to cure the problem we consider the function

$$
\varphi_{i j}(z)=r_{i j}(z, w)+\frac{\delta_{i j}}{w-z},
$$

which is already holomorphic at the point $z=w$, and therefore the Laurent expansion

$$
\begin{gathered}
\left.\varphi_{i j}(z)=-\frac{\alpha_{a}^{i} u_{a j}(w) d w}{z-\gamma_{a}}+\left(r_{i j}^{a, 0}(w)\right)+\frac{\delta_{i j}}{w-\gamma_{a}}\right)+ \\
+\left(r_{i j}^{a, 1}(w)+\frac{\delta_{i j}}{\left(w-\gamma_{a}\right)^{2}}\right)\left(z-\gamma_{a}\right)+o\left(z-\gamma_{a}\right),
\end{gathered}
$$

of the function is convergent on the neighborhood $U_{\gamma_{a}}$ even in the case when the point $w$ is on the neighborhood. 
Hence, we can apply the remaining properties of the differential $r_{i j}(z, w) d w$ to expansion (32) and finally get that on the neighborhood $U_{\gamma_{a}}$ the differentials $r_{i j}^{a, 0}(w) d w$ and $r_{i j}^{a, 1}(w) d w$ behave like

$$
\begin{gathered}
r_{i j}^{a, 0}(w) d w=-\frac{\delta_{i j}}{w-\gamma_{a}}+\text { regular terms, } \\
r_{i j}^{a, 1}(w) d w=-\frac{\delta_{i j}}{\left(w-\gamma_{a}\right)^{2}}+\text { regular terms, }
\end{gathered}
$$

and $\alpha_{a}$ is a right null vector for the regular parts of the matrices $\left\|r_{i j}^{a, 0}(w)\right\|$ and $\left\|r_{i j}^{a, 1}(w)\right\|$ at the point $w=\gamma_{a}$.

Thus, the lemma is proved.

\subsection{Derivatives of the Krichever Lax differential.}

In this subsection we present the properties of derivatives of the differential (9) with respect to the variables $\gamma_{a}$ and $\kappa_{a}$ and with respect to the canonical coordinates $\alpha_{a}^{\mu}$ and $\beta_{a}^{\mu} \mu=2, \ldots, n$ in the local chart (24) on the space $\mathcal{P}$. As it will be seen the properties uniquely define the derivatives of $L$ as meromorphic differentials on the curve $\Sigma$. form

First, we note that the differential $\partial_{k_{a}} L_{i j}(z) d z$ can be written in the following

$$
\partial_{k_{a}} L_{i j}(z) d z=\alpha_{a}^{j} u_{a i}(z) d z,
$$

where $u_{a i}(z) d z$ are holomorphic differentials defined by equations (27).

Second, the differential $\partial_{\beta_{a}^{\mu}} L_{i j}(z) d z$ has at most simple poles at the points $P$ and $\gamma_{a}$ and the residue of $\partial_{\beta_{a}^{\mu}} L_{i j}(z) d z$ at the point $\gamma_{a}$ equals

$$
\operatorname{Res}_{z=\gamma_{a}} \partial_{\beta_{a}^{\mu}} L_{i j}(z) d z=\delta_{i \mu} \alpha_{a}^{j}-\delta_{i 1} \alpha_{a}^{\mu} \alpha_{a}^{j} .
$$

For $b \neq a, \alpha_{b}$ is a left null vector for the matrix $\left\|\partial_{\beta_{a}^{\mu}} L_{i j}\left(\gamma_{b}\right)\right\|$

$$
\sum_{i=1}^{n} \alpha_{b}^{i} \partial_{\beta_{a}^{\mu}} L_{i j}\left(\gamma_{b}\right)=0, \quad b \neq a,
$$

and $\alpha_{a}$ is a left null vector for the regular part of the matrix $\left\|\partial_{\beta_{a}^{\mu}} L_{i j}(z)\right\|$ at point $\gamma_{a}$ :

$$
\left.\sum_{i=1}^{n} \alpha_{a}^{i} \partial_{\beta_{a}^{\mu}} L_{i j}(z)\right|_{\text {regular part at } z=\gamma_{a}}=0 .
$$

Third, the differential $\partial_{\alpha_{a}^{\mu}} L_{i j}(z) d z$ also has at most simple poles at the points $P$ and $\gamma_{a}$ and the residue of $\partial_{\alpha_{a}^{\mu}} L_{i j}(z) d z$ at the point $\gamma_{a}$ equals

$$
\operatorname{Res}_{z=\gamma_{a}} \partial_{\alpha_{a}^{\mu}} L_{i j}(z) d z=\beta_{a}^{i} \delta_{j \mu}-\delta_{i 1} \beta_{a}^{\mu} \alpha_{a}^{j}
$$


For $b \neq a, \alpha_{b}$ is a left null vector for the matrix $\left\|\partial_{\alpha_{a}^{\mu}} L_{i j}\left(\gamma_{b}\right)\right\|$

$$
\sum_{i=1}^{n} \alpha_{b}^{i} \partial_{\alpha_{a}^{\mu}} L_{i j}\left(\gamma_{b}\right)=0, \quad b \neq a
$$

and the regular part the matrix $\left\|\partial_{\alpha_{a}^{\mu}} L_{i j}(z)\right\|$ at point $\gamma_{a}$ satisfies the following linear inhomogeneous equation (for the definition of the matrix $\left\|L_{i j}^{a, 0}\right\|$ see equation (9))

$$
\left.\sum_{i=1}^{n} \alpha_{a}^{i} \partial_{\alpha_{a}^{\mu}} L_{i j}(z)\right|_{\text {regular part at } z=\gamma_{a}}=\left(k_{a} \delta_{\mu j}-L_{\mu j}^{a, 0}\right) .
$$

Finally, the differential $\partial_{\gamma_{a}} L_{i j}(z) d z$ is holomorphic everywhere on $\Sigma$ except the point $\gamma_{a}$, where it has a pole of the second order and on a neighborhood of the point it behaves like

$$
\partial_{\gamma_{a}} L_{i j}(z) d z=\frac{\beta_{a}^{i} \alpha_{a}^{j} d z}{\left(z-\gamma_{a}\right)^{2}}+\text { regular terms. }
$$

For $b \neq a, \alpha_{b}$ is a left null vector for the matrix $\left\|\partial_{\gamma_{a}} L_{i j}\left(\gamma_{b}\right)\right\|$

$$
\sum_{i=1}^{n} \alpha_{b}^{i} \partial_{\gamma_{a}} L_{i j}\left(\gamma_{b}\right)=0, \quad b \neq a
$$

and, in addition, the regular part of the matrix $\left\|\partial_{\gamma_{a}} L_{i j}(z)\right\|$ at the point $\gamma_{a}$ satisfies the following linear inhomogeneous equation (for the definition of the matrix $\left\|L_{i j}^{a, 1}\right\|$ see equation (9))

$$
\left.\sum_{i=1}^{n} \alpha_{a}^{i} \partial_{\gamma_{a}} L_{i j}(z)\right|_{\text {regular part at } z=\gamma_{a}}=-\sum_{i=1}^{n} \alpha_{a}^{i} L_{i j}^{a, 1} .
$$

All the properties of the derivatives $\partial_{k_{a}} L_{i j}(z) d z, \partial_{\gamma_{a}} L_{i j}(z) d z, \partial_{\alpha_{a}^{\mu}} L_{i j}(z) d z$ and $\partial_{\beta_{a}^{\mu}} L_{i j}(z) d z$ can be easily derived from the definition of the Krichever Lax differential (9) and the uniqueness of the derivatives as meromorphic differentials on $\Sigma$ follows directly from the Krichever lemma.

\subsection{The sketch of the proof.}

Let us rewrite the Yang-Baxter relation (13) in the following form

$$
D_{i j k l}(z, w) d z \otimes d w=R_{i j k l}(z, w) d z \otimes d w
$$

where

$$
\begin{gathered}
D_{i j k l}(z, w)=\left\{L_{i j}(z), L_{k l}(w)\right\}= \\
=\sum_{a=1}^{n g}\left(\partial_{\gamma_{a}} L_{i j}(z) \partial_{k_{a}} L_{k l}(w)-\partial_{k_{a}} L_{i j}(z) \partial_{\gamma_{a}} L_{k l}(w)\right)+ \\
+\sum_{a=1}^{n g} \sum_{\mu=2}^{n}\left(\partial_{\alpha_{a}^{\mu}} L_{i j}(z) \partial_{\beta_{a}^{\mu}} L_{k l}(w)-\partial_{\beta_{a}^{\mu}} L_{i j}(z) \partial_{\alpha_{a}^{\mu}} L_{k l}(w)\right),
\end{gathered}
$$


and

$$
\begin{gathered}
R_{i j k l}(z, w)=\sum_{m=1}^{n} \delta_{i l} r_{m k}(z, w) L_{m j}(z)-L_{i l}(z) r_{j k}(z, w)- \\
-\sum_{m=1}^{n} \delta_{k j} r_{m i}(w, z) L_{m l}(w)+L_{k j}(w) r_{l i}(w, z) .
\end{gathered}
$$

Using the properties of the differentials $\partial_{k_{a}} L_{i j}(z) d z, \partial_{\gamma_{a}} L_{i j}(z) d z, \partial_{\alpha_{a}^{\mu}} L_{i j}(z) d z$ and $\partial_{\beta_{a}^{\mu}} L_{i j}(z) d z$ we derive a relatively long list of properties for the form $D_{i j k l}(z, w) d z \otimes d w$ :

1. The poles of the form $D_{i j k l}(z, w) d z \otimes d w$ are located at the points $\gamma_{a}$ and $P$ so that the pole at the point $P$ is simple and the poles at the points $\gamma_{a}$ are of the second order.

2. If $w$ coincides neither with the point $P$ nor with any of the points $\gamma_{b}$ the singular part of the component $D_{i j k l}(z, w)$ at the point $z=\gamma_{a}$ looks like

$$
D_{i j k l}(z, w)=\frac{D_{i j k l}^{a, 2}(w)}{\left(z-\gamma_{a}\right)^{2}}+\frac{D_{i j k l}^{a, 1}(w)}{z-\gamma_{a}}+\text { regular terms, }
$$

where $D_{i j k l}^{a, 2}(w)$ is a component of the holomorphic differential $\alpha_{a}^{l} \beta_{a}^{i} \alpha_{a}^{j} u_{a k}(w) d w$ and $D_{i j k l}^{a, 1}(w) d w$ is a differential of the third kind defined by the following properties

- $D_{i j k l}^{a, 1}(w) d w$ has poles only at the points $\gamma_{a}$ and $P$ with the residue at the point $\gamma_{a}$ being

$$
\operatorname{Res}_{w=\gamma_{a}} D_{i j k l}^{a, 1}(w) d w=\delta_{k j} \beta_{a}^{i} \alpha_{a}^{l}-\delta_{i l} \beta_{a}^{k} \alpha_{a}^{j} .
$$

- The values of the components $D_{i j k l}^{a, 1}(w)$ at the points $\gamma_{b}, b \neq a$ satisfy the following "null vector" conditions

$$
\sum_{k=1}^{n} \alpha_{b}^{k} D_{i j k l}^{a, 1}(w)=0, \quad b \neq a .
$$

- The regular parts of $D_{i j k l}^{a, 1}(w)$ at the point $\gamma_{a}$ obey the following linear inhomogeneous equations

$$
\left.\sum_{k=1}^{n} \alpha_{a}^{k} D_{i j k l}^{a, 1}(w)\right|_{\text {regular part at } w=\gamma_{a}}=-\left(k_{a} \delta_{i l}-L_{i l}^{a, 0}\right) \alpha_{a}^{j} .
$$

3. The regular parts of the components $D_{i j k l}(z, w)$ at the points $\gamma_{a}$ satisfy the linear inhomogeneous equations

$$
\left.\sum_{i=1}^{n} \alpha_{a}^{i} D_{i j k l}(z, w)\right|_{\text {regular part at } z=\gamma_{a}}=D_{j k l}^{a}(w),
$$

where $D_{j k l}^{a}(w)$ are components of a meromorphic tensor-valued differential defined by the following properties 
- $D_{j k l}^{a}(w) d w$ is holomorphic everywhere on $\Sigma$ except the points $P$ and $\gamma_{a}$ where it has poles of the first and second order respectively.

- On a neighborhood of the point $w=\gamma_{a}$ it behaves like

$$
D_{j k l}^{a}(w) d w=-\frac{\beta_{a}^{k} \alpha_{a}^{l} \alpha_{a}^{j} d w}{\left(w-\gamma_{a}\right)^{2}}+\frac{\left(k_{a} \delta_{k j}-L_{k j}^{a, 0}\right) \alpha_{a}^{l} d w}{w-\gamma_{a}}+\text { regular terms. }
$$

- The values of the components $D_{j k l}^{a}(w)$ at the points $\gamma_{b}, b \neq a$ satisfy the following "null vector" conditions

$$
\sum_{k=1}^{n} \alpha_{b}^{k} D_{j k l}^{a}\left(\gamma_{b}\right)=0, \quad b \neq a .
$$

- The regular parts of the components $D_{j k l}^{a}(w)$ at the point $\gamma_{a}$ obey the following linear inhomogeneous equations

$$
\left.\sum_{k=1}^{n} \alpha_{a}^{k} D_{j k l}^{a}(w)\right|_{\text {regular part at } w=\gamma_{a}}=\sum_{k=1}^{n}\left(\alpha_{a}^{j} \alpha_{a}^{k} L_{k l}^{a, 1}-\alpha_{a}^{l} \alpha_{a}^{k} L_{k j}^{a, 1}\right) .
$$

An analogous detailed analysis of the components $R_{i j k l}(z, w)$ shows that $R_{i j k l}(z, w) d z \otimes$ $d w$ satisfies all the above properties of the form $D_{i j k l}(z, w) d z \otimes d w$. Due to the Krichever lemma these properties define a unique form $D_{i j k l}(z, w) d z \otimes d w$ and, thus, the desired statement is proved.

\section{Concluding remarks.}

In conclusion, we point out that the classical $r$-matrix (14) of the extended Krichever Lax matrix (9) depends only on the variables $\gamma_{a}$ and $\alpha_{a}$, that is, on coordinates of the respective configuration space. Since the differential (9) is linear in the variables $k_{a}$ and $\beta_{a}^{i}$, the genuine $r$-matrix (20) of the Hitchin system also depends only on the variables $\gamma_{a}$ and $\alpha_{a}$.

This forces us to assume that the classical $r$-matrices satisfy simple analogues of classical dynamical Yang-Baxter equation [11], which should express the consistency of the respective Yang-Baxter relations for the Krichever Lax matrices (9) and (16).

Note also that a formal expression for the classical $r$-matrix of the extended system can be obtained by the method developed in the paper [5]. Following that method we have to present the system on the manifold (6) with the Krichever Lax matrix (9) via an infinite-dimensional Hamiltonian reduction on $n g$ copies of the cotangent bundle to the loop group $G L_{n}(\mathbb{C})\left[z, z^{-1}\right]$. Although the method allows one to express the desired $r$-matrix in terms of infinite series in the Krichever-Novikov type basis [23], [29], [31] it turns out to be very hard to analyze such answers and to identify the resulting $r$-matrix with any meromorphic object associated with the product of curves $\Sigma \times \Sigma$.

Finally, we mention that it would be interesting to compare the Krichever parameterization of Lax and $r$-matrix structures of Hitchin systems based on Tyurin 
description to the analogous approach [9] based on the Schottky uniformisation of Riemann curves and it would be also intriguing to explain a role of the obtained $r$-matrices in the context of WZNW models on Riemann surface [6], [17].

Acknowledgements. I would like to express my sincere thanks to I.M. Krichever and M.A. Olshanetsky for formulating the problem and for useful discussions of this topic. I acknowledge I.M. Krichever for constructive criticisms concerning the first version of this article and A.M. Levin for an important technical trick, which drastically simplifies the result of this paper. I also acknowledge H.W. Braden, A.S. Gorsky, S.V. Oblezin and A.V. Zotov for useful discussions. I am grateful to M. Ching for criticisms concerning the English language of this paper. The work is partially supported by RFBR grant 00-02-17-956, the Grant for Support of Scientific Schools 00-15-96557, and the grant INTAS 00-561.

\section{Appendix. The proof of the Krichever lemma.}

Lemma 5 (Krichever) Let $\nu_{i}(z) d z$ be a meromorphic vector-valued differential on the curve $\Sigma$. Then, for a generic set of Tyurin parameters $\gamma_{a} \in \Sigma$ and $\alpha_{a} \in \mathbb{C}^{n}$ and for an arbitrary set of complex numbers $b_{a}$ there exists a unique meromorphic vectorvalued differential $v_{i}(z) d z$ having the same singular parts as the differential $\nu_{i}(z) d z$ and obeying the following conditions ${ }^{8}$ :

- If $v_{i}(z) d z$ is holomorphic at the point $\gamma_{a}$ then

$$
\sum_{i=1}^{n} v_{i}\left(\gamma_{a}\right) \alpha_{a}^{i}=b_{a}
$$

- and otherwise,

$$
\left.\sum_{i=1}^{n} v_{i}(z) \alpha_{a}^{i}\right|_{\text {regular part at } \mathrm{z}=\gamma_{\mathrm{a}}}=b_{a} .
$$

Proof. The statement of the lemma is equivalent to the fact that for a generic set of Tyurin parameters $\left(\gamma_{a}, \alpha_{a}\right)$ and for an arbitrary set of complex numbers $c_{a}$ there exists a unique holomorphic vector-valued differential $h_{i}(z) d z$ satisfying the equations

$$
\sum_{i=1}^{n} h_{i}\left(\gamma_{a}\right) \alpha_{a}^{i}=c_{a}
$$

which are, in turn, equivalent to the following linear inhomogeneous equations

$$
\sum_{i=1}^{n} \sum_{A=1}^{g} h_{i}^{A} \mu_{A}\left(\gamma_{a}\right) \alpha_{a}^{i}=c_{a}
$$

\footnotetext{
${ }^{8}$ Note that we choose some local coordinates on neighborhoods of the points $\gamma_{a}$ and the right hand sides of equations (46) and (47) depend on this choice
} 
for the expansion coefficients $h_{i}^{A}$ of the differential $h_{i}(z) d z$ in some basis $\left\{\mu_{A}(z) d z, A=\right.$ $1, \ldots, g\}$ of holomorphic differentials on the curve $\Sigma$.

Since the number of coefficients $h_{i}^{A}$ coincides with the number of equations (49) the desired statement is equivalent to the fact that the following $n g \times n g$-matrix

$$
M_{a}^{(A i)}=\mu_{A}\left(\gamma_{a}\right) \alpha_{a}^{i}
$$

is non-degenerate.

The proof of this fact turns out to be a simple task of linear algebra. $\square$

\section{References}

[1] Arutyunov G.E., Medvedev P.B.: Geometric construction of the classical $R$-matrices for the elliptic and trigonometric Calogero-Moser systems. hepth/9511070

[2] Avan J., Babelon O. and Talon M.: Construction of the classical $R$-matrices for the Toda and Calogero models. Algebra i Analiz 6, 2, 67-89 (1994); PARLPTHE-93-31, hep-th/9306102

[3] Babelon O., Viallet C.M.: Hamiltonian Structures and Lax Equation. Phys. Lett. B 237, 411-416 (1990)

[4] Beilinson A.A., Drinfeld V.G.: Quantization of Hitchin's fibration and Langlands program. Preprint, 3 pp., 1993; Laumon G.: Correspondance de Langlands geometrique pour les corps de fonctions. Duke Math. J. 54, 309-359 (1987)

[5] Braden H.W., Dolgushev V.A., Olshanetsky M.A. and Zotov A.V.: Classical $R$-matrices and Feigin-Odesskii algebra via Hamiltonian and Poisson reductions. hep-th/0301121.

[6] Bernard D.: On the Wess-Zumino-Witten models on torus. Nucl. Phys. B303, 77-93 (1988); On the Wess-Zumino-Witten models on Riemann surfaces. Nucl. Phys. B309, 145-174 (1988)

[7] Enriquez B., Rubtsov V.: Hitchin systems, higher Gaudin operators and $R$ matrices. Math. Res. Lett. 3, 3, 343-357 (1996)

[8] Enriquez B., Rubtsov V.: Hecke-Tyurin parametrization of the Hitchin and KZB systems. math.AG/9911087

[9] Enriquez B.: Dynamical r-matrices for Hitchin's systems on Schottky curves. Lett. Math. Phys. 45, 2, 95-104 (1998)

[10] Etingof P., Varchenko A.: Solutions of the quantum dynamical Yang-Baxter equation and dynamical quantum groups. Commun. Math. Phys. 196, 3, 591640 (1998)

[11] Etingof P., Varchenko A.: Geometry and classification of solutions of the classical dynamical Yang-Baxter equation. Commun. Math. Phys. 192, 1, 77-120 (1998) 
[12] Faddeev L.D., Takhtajan L.A.: Hamiltonian methods in the theory of solitons. Springer Series in Soviet Mathematics. Berlin: Springer-Verlag, 1987.

[13] Fehér L., Gábor A. and Pusztai B.G.: On dynamical r-matrices obtained from Dirac reduction and their generalizations to affine Lie algebras. J. Phys. A, Math. Gen. 34, 36, 7335-7348 (2001)

[14] Feigin B., Frenkel E. and Reshetikhin N.: Gaudin model, Bethe ansatz and critical level. Commun. Math. Phys. 166, 1, 27-62 (1994)

[15] Felder G.: Elliptic quantum groups. In: D. Iagolnitzer. (ed.) Mathematical Physics . Proceedings. Paris 1994, pp. 211-218. Cambridge, USA: IP, 1995

[16] Felder G.: Conformal field theory and integrable systems associated to elliptic curves. Proceedings of the International Congress of Mathematicians, Vol. 1, 1247-1255, Birkhuser, Basel, 1995.

[17] Felder G.: The KZB equations on Riemann surfaces. Quantum symmetries. Proceedings. Les Houches 1995, pp. 687-725

[18] Gervais J.-L., Neveu A.: Novel triangle relation and absence of tachyons in Liouville string field theory. Nucl. Phys. B238, 125-141 (1984)

[19] Grinevich P.: Rational solutions for the equations of commutation of differential operators. Funct. Anal. Appl. 16, 19-24 (1982)

[20] Hitchin N.: Stable Bundles and Integrable Systems. Duke Math. J. 54, 1, 91-114 (1987)

[21] Krichever I.M.: Vector bundles and Lax equations on algebraic curves. Commun. Math. Phys. 229, 2, 229-269 (2002); hep-th/0108110

[22] Krichever I.M., Novikov S.P.: Holomorphic bundles over Riemann surfaces and the KP equations, I. Funct. Anal. Appl. 12, 41-52 (1978)

[23] Krichever I.M., Novikov S.P.: Algebras of Virasoro type, Riemann surfaces and structures of the theory of solitons. Funct. Anal. Appl. 21, 126-142 (1987);

Virasoro-type algebras, Riemann surfaces and strings in Minkowski space. Funct. Anal. Appl. 21, 294-307 (1987);

Virasoro-Gelfand-Fuks type algebras, Riemann surfaces, operator's theory of closed strings. J. Geom. Phys. 5, 4, 631-661 (1988);

Algebras of Virasoro type, energy-momentum tensor, and decomposition operators on Riemann surfaces. Funct. Anal. Appl. 23, 19-33 (1989)

[24] Levin A.M., Olshanetsky, M.A. and Zotov A.V.: Hitchin systems - symplectic Hecke correspondence and two-dimensional version. ITEP-TH-56-01, nlin.si/0110045

[25] Markman, E.: Spectral curves and integrable systems. Compositio Math. 93, 3, 255-290 (1994) 
[26] Nekrasov N.: Commun. Math. Phys. 180, 587-604 (1996)

[27] Olshanetsky M.A.: Lett. Math. Phys. 42, 59-71 (1997)

[28] Previato E., Wilson G.: Vector bundles over curves and solutions of the KP equations. In Theta functions. Proc. Symp. Pure Math. AMS Bowdoin, 1987, 49, 1, pp. 553-570

[29] Schlichenmaier M., Sheinman O. K.: The Wess-Zumino-Witten-Novikov theory, Knizhnik-Zamolodchikov equations, and Krichever-Novikov algebras, I, Mannheimer Manuskripte 236, math.QA/9812083

[30] Semenov-Tian-Shansky M.A.: What is a classical r-matrix? Funct. Anal. Appl. 17, 259-272 (Russian) (1983); 17-33 (English translation).

[31] Sheinman O.K.: Elliptic affine Lie algebras. Funct. Anal. Appl. 24, 3, 210-219 (1990);

Highest weight modules over certain quasigraded Lie algebras on elliptic curves. Funct. Anal. Appl. 26, 3, 65-71 (1992);

Affine Lie algebras on Riemann surfaces. Funct. Anal. Appl. 27, 4, 54-62 (1993); Highest weight modules for affine Lie algebras on Riemann surfaces. Funct. Anal. Appl. 29, 1, 56-71 (1995)

[32] Sklyanin E.K.: On the complete integrability of the Landau-Lifchitz equation. Preprint LOMI E-3-79. Leningrad 1979.

[33] Talalaev D.: The elliptic Gaudin system with spin. Theor. Math. Phys. 130, 361-374 (2002)

[34] Tyurin A.: Classification of vector bundles over an algebraic curve of arbitrary genus. Am. Math. Soc., Translat., II. Ser. 63, 245-279 (1967) 